Supplemental Information for

\title{
Stretching of tethered DNA in nanoslits
}

\author{
Jia-Wei Yeh*, Kylan Szeto \\ School of Applied and Engineering Physics, Cornell University, Ithaca, NY, 14853, USA
}

\section{Materials and methods}

Micro/nano-fluidic channel fabrication. The micro/nano-fluidic channels were fabricated on fused silica substrates using two steps of photolithography and reactive ion etching. The nanoslit pattern consisted of 50 parallel nanoslit channels, with length $l_{s}=30$ (used for $\lambda$-DNA stretching), and $90 \mu \mathrm{m}$ (used for chromatin stretching), width $w_{s}=10 \mu \mathrm{m}$, and heights $h=30,40$, $55,60,130 \mathrm{~nm}$. The microchannel pattern was defined approximately $1 \mu \mathrm{m}$ in depth and $184 \mu \mathrm{m}$ in width. The device input and output holes were made using a sand bluster, cleaned by Piranha etching and RCA cleaning, and finally touch bonded with $170 \mu \mathrm{m}$-thick fused silica wafer to enclose the fluidic channels. The wafer stack was annealed at $1080{ }^{\circ} \mathrm{C}$. Sample loading reservoirs were attached to the substrate by fast-curing epoxy. The electrical contacts were made by inserting gold electrodes into each of the reservoirs. A schematic of the device is shown in Figure 1c and S1.

DNA-bead sample preparation. $\lambda$-DNA $[48.5 \mathrm{~kb}$, from New England Biolabs (NEB)] with one end labeled with biotin, was attached to streptavidin-coated polystyrene beads [diameter 0.2 
$\mu \mathrm{m}$ (green fluorescent) or $0.4 \mu \mathrm{m}$ (red fluorescent)] (Bangs's Laboratories, Inc). The beads were made by covalently conjugating Streptavidin to fluorescent carboxyly-modified polystyrene beads. The uncoated carboxyl-modified polystyrene beads used for coating with Streptavdin likely had negative-charged surface due to the presence of bead surface carboxyl and sulfate groups. Therefor, the bead would run the same direction with DNA, stuck at micro/nano entrance, and contribute no force to our system. The procedure to attach biotin to one end of the $\lambda$-DNA consisted of the hybridization and subsequent ligation of a specially designed primer to the cos site of lambda DNA. The primer sequence was 5'- GGGCGGCGACCT-3'-B (Integrated DNA Technologies, IDT), which contained a single biotin label (B) at the 3' end. For the hybridization, $20 \mu \mathrm{l}$ of $500 \mu \mathrm{g} / \mathrm{ml}$ DNA was mixed with $28 \mu \mathrm{l}$ of primer $(100 \mathrm{mM})$ in the present of ligase buffer (10X T4 Ligase Reaction buffer, NEB) in a $145 \mu l$ reaction volume. The mixture was incubated at $65{ }^{\circ} \mathrm{C}$ for an hour and then left at room temperature for at least 2 hours. DNA ligase was carried out by adding $5 \mu \mathrm{l}$ of 20,000 units T4 DNA ligase (NEB) and incubated overnight at $16{ }^{\circ} \mathrm{C}$. Following the ligation, a phenol-chloroform extraction was performed to remove the remaining enzymes and primers. The DNA was then resuspended in $10 \mathrm{mM}$ TrisEDTA buffer at $\mathrm{pH}$ 8.0.

After washing beads in $0.1 \%$ Tween-20, $0.1 \mathrm{M} \mathrm{NaCl}, 10 \mathrm{mM}$ Tris-HCl $1 \mathrm{mM}$ EDTA (TE) buffer at $\mathrm{pH}=8.0$, DNA was mixed with beads at a number ratio of 1:1. DNA was then stained with YOYO-1 (Invitrogen) at a dye-to-basepair ratio of 1:5 in imaging buffer containing $2.5 \%$ (w/w) poly(n-vinylpyrrolidone) (PVP, K30, wt 40000, Sigma), used to suppress electro-osmotic flow, $30 \%$ (w/v) sucrose, $0.1 \%$ Tween 20, TE buffer (10 mM Tris-HCl 1 mM EDTA) and an oxygen scavenging system including $50 \mu \mathrm{g} / \mathrm{ml}$ glucose oxidase (Sigma), $10 \mu \mathrm{g} / \mathrm{ml}$ catalase (Sigma), $0.1 \%$ DTT, and $0.5 \%$ (v/v) 2-mercaptoethanol (BME, Sigma). A Debye length of 1.6 
nm was calculated from the ionic strength $\left(I=4.2 \times 10^{-2} \mathrm{M}\right)$ of our buffer condition, which is much smaller than the slit height; therefore electro-osmotic flow was not taken into account in our system.

Microscopy and image analysis. Single DNA molecules were observed in a fluorescence microscopy system consisting of an inverted microscope (Olympus), a 100X oil-immersion lens (Leica, N.A. 1.35), and an EMCCD camera (IXon-897, Andor Technologies) with an equivalent pixel resolution of $160 \mathrm{~nm}$. Images were captured with an exposure time of $0.1 \mathrm{sec}$. DNA were analyzed from the CCD images using ImageJ (NIH) software and MATLAB (The Mathworks, Natick, MA). An automated algorithm was developed to track the fluorescence peaks by Gaussian fitting through the entire DNA polymer with pixel-step. This method enabled us to determine the contour trajectory and extension length of DNA confined in the nanoslit.

\section{Calculation of electric field in the nanoslit}

The resistance along the microchannel $R_{\mu}$ is $\varrho_{b} L_{\mu} / h_{\mu} w_{\mu}$, with $h_{\mu}$ the depth of microchannels ( 1 $\mu \mathrm{m}), w_{\mu}$ the width of microchannel $(184 \mu \mathrm{m})$ and $L_{\mu}$ the length of microchannel from the reservoirs to the nanoslit (5.95 mm). The nanoslit resistance $R_{n}$ of the array is $\varrho_{b} l_{s} / h w_{s} n$ with $n$ the number of nanochannels (50), $h, l_{s}$, and $w_{s}$ are the nanoslit height, length $(30 \mu \mathrm{m})$, and width (10 $\mu \mathrm{m})$. Therefore, $R_{n} / R_{\mu}=0.05,0.033,0.015$ for $h=40,60$, and $130 \mathrm{~nm}$ slits, respectively. The voltage ratio for the nanoslits and the microchannel is proportional to resistance, $V_{n} / V_{\mu}=R_{n} / R_{\mu}$. According to the circuit model of the chip, the applied voltage, $V$, is equal to $2 V_{\mu}+V_{n}$. Therefore, $V_{n} / V=0.024,0.016,0.0077$ for $h=40,60$, and $130 \mathrm{~nm}$ slits, respectively. We can further estimate the electric field across the nanoslit $E_{n}=V_{n} / l_{s}$. Therefore, $E_{n}=8.2,5.5,2.5($ Volts $/ \mathrm{cm})$ multiplied by the applied voltage $V$ for $h=40,60$, and $130 \mathrm{~nm}$ slits. 
A recent study ${ }^{1}$ points out that there can be additional resistances, $R_{f f}=\varrho_{b} f / L_{\mu}$ due to the focusing of the electric field at the interface which can affect the calculation of the electric field in different parts of the device. In our system, $\frac{R_{f f}}{R_{\mu}}=\frac{\bar{f} h_{\mu} w_{\mu}}{L_{\mu}{ }^{2}} \approx 5 \times 10^{-6} \bar{f} \approx 10^{-4}$, which indicates that the interfacial resistance $R_{f f}<R_{n} \ll R_{\mu}$ is negligible in our case.

\section{Force-extension relation of tethered end stretched polymer}

The force-extension relation for a DNA polymer in free solution has been measured by applying a force at both ends with optical tweezers or magnetic tweezers ${ }^{2}$, or by applying a force along the whole chain with electrophoretic or hydrodynamic flow ${ }^{3,4}$. Marko and Siggia 5 developed the WLC force-extension relations for both methods of stretching. Their forceextension relation for applying a force at two ends that closely approximates the conditions of the experiments in references ${ }^{2,3,6}$, is given by

$$
f_{e l s}=\frac{k_{B} T}{l_{p}}\left[\frac{1}{4}\left(\frac{1}{(1-x)^{2}}-1\right)+x\right]
$$

where $k_{B} T$ is the thermal energy, $f_{e l s}$ is the elastic force, $l_{p}$ is the persistence length, and $x=l / L$ is the relative extension of the chain. This expression captures the linear dependence for small extensions and non-linear behavior for large extensions. It is well-suited for describing long free chains stretched by applying forces at both ends.

For stretching a single end-tethered polymer with an electric field $\boldsymbol{E}=E \hat{x}$, the force is characterized by an inhomogeneous stretching along the polymer. The tension at a point $s$ on a chain anchored at point $s=0$ is 


$$
f_{e}(s)=\lambda E \int_{s}^{L} \hat{t} \cdot \hat{x} d s
$$

where $\hat{t}$ is unit tangent vector. The tension decreases along the chain from an anchored point to its free end. The stored electric energy $\varepsilon_{E}$ can be calculated by integration of eq S2:

$$
\varepsilon_{E}=\lambda E \int_{0}^{L} \hat{t} \cdot \hat{x}\left(\int_{s}^{L} \hat{t} \cdot \hat{x} d s\right) d s
$$

According to the balance of the averaged $f_{e}$ of eq S2 and $f_{e l s}$ of eq S1, the tangent vector orientation profiles $y(s) \equiv\langle\hat{y} \cdot \hat{t}\rangle$ can be characterized ${ }^{5}$ by:

$$
\left[1+\frac{1}{2(1-y)^{3}}\right] \frac{d y}{d s}=-\varepsilon \frac{y}{A}
$$

where the dimensionless electric field is $\varepsilon \equiv \lambda E l_{p}^{2} / k_{B} T$, and $\lambda$ is the effective charge density per unit length, which is assumed to be a uniform distribution along chain due to total HI screening in strong confinement. Thanks to Monte Caro Simulations from ref ${ }^{7}$, the approximate forceextension expression in 3D and 2D case is given by:

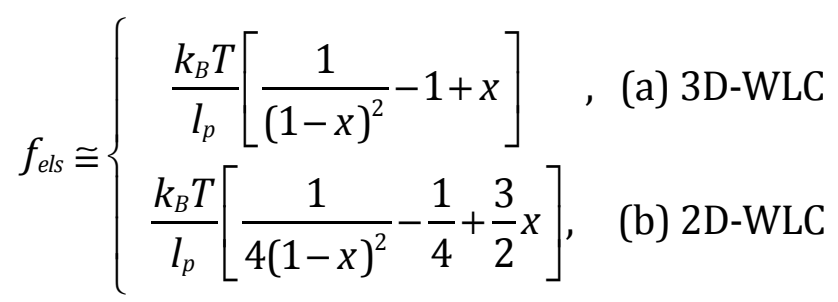

\section{Stretching tethered-DNA in $30 \mathrm{~nm}$ nanoslits}

In order to verify how surface interactions affect the stretching mechanism of biopolymers, we further reduced the slit height to $h=30 \mathrm{~nm}$, which is smaller than the DNA persistence length but still much larger than the Debye screening length $\sim 2.27 \mathrm{~nm}$. In this case, the highly confined 
DNA demonstrated a reptation trajectory that was qualitatively different from the contour shapes in slits that were higher than $40 \mathrm{~nm}$. This reptation motion was also observed when the DNA was retracted by purely entropic forces in a similar highly-confined environment ${ }^{8}$. The diffusivities of DNA measured along the $\mathrm{x}$ - and $\mathrm{y}$ - axis were observed to have significant differences ${ }^{9}$. We stretched and relaxed the DNA by switching the applied voltage across the nanoslits between $0.03 \mathrm{~V}$ (stretch) and $-0.03 \mathrm{~V}$ (retract) repeatedly (Movie S3). Some representative DNA contours are shown in Figure S2a. To understand the statistics and variation of the elongated DNA contours, we overlaid 70 images of different extensions of the same molecule to illustrate the angular spread of the DNA molecule trajectories (Figure S2b) and we traced their contours individually for further analysis (Figure S2c). The traced DNA trajectories allowed calculations of the forces by eq S2 and stored electric energy distributions due to the applied fields by eq S3. The approximately linear decay of the force-position relation (Figure S2d) is in agreement with previous theoretical work ${ }^{5}$. We also found that the stored electric energy $\varepsilon_{E}$ due to the applied field had a wide probability distribution $P\left(\varepsilon_{E}\right)$, which cannot be attributed to thermal fluctuations $\left(k_{B} T\right)$ (Figure S2e). The broad probability distribution and the fact that the DNA is in equilibrium suggest the existence of an additional contribution to the system's overall energy. This additional energy may arise from strong charge interactions between the DNA and the glass walls of the nanoslit. Moreover, the snake-like trajectory of the DNA (Figure S2b) implies that the scale of the surface inhomogeneity of the nanoslit walls and its surface passivating coatings are on the order of microns. The extra DNA extension in this regime may be explained by taking into account both the effective width of DNA $\left(w_{d} \sim 5.5 \mathrm{~nm}\right){ }^{10}$ and the deposited Polyvinylpyrrolidone (PVP) on the surface $\left(w_{p} \sim 4 \mathrm{~nm}\right)^{8,11}$; therefore, the effective slit height can be described as $h_{e}=h$ - $2\left(w_{d}+w_{p}\right)$ where $h_{e}$ is $\sim 11 \mathrm{~nm}$ for $30 \mathrm{~nm}$ nanoslits. 


\section{Figures}

(a)

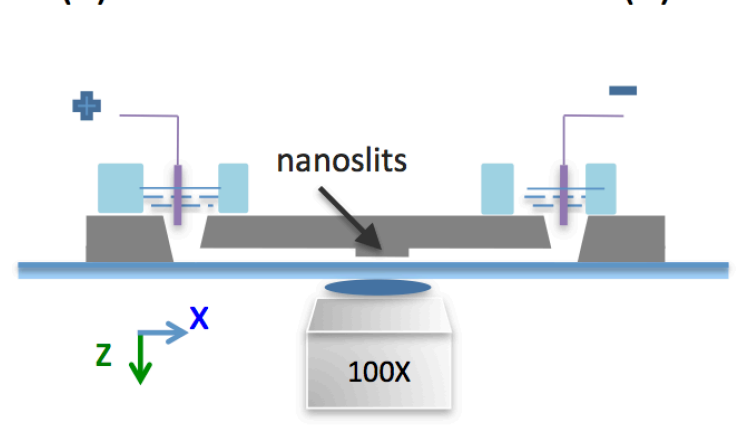

(b)

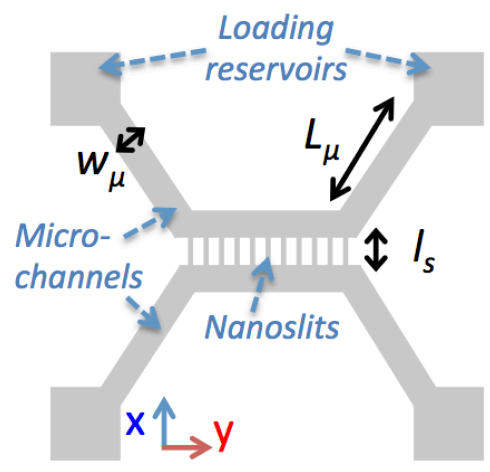

Figure S1 (a) Side view of the device and imaging setup. (b) Top view of the microchannel and nanoslit pattern.
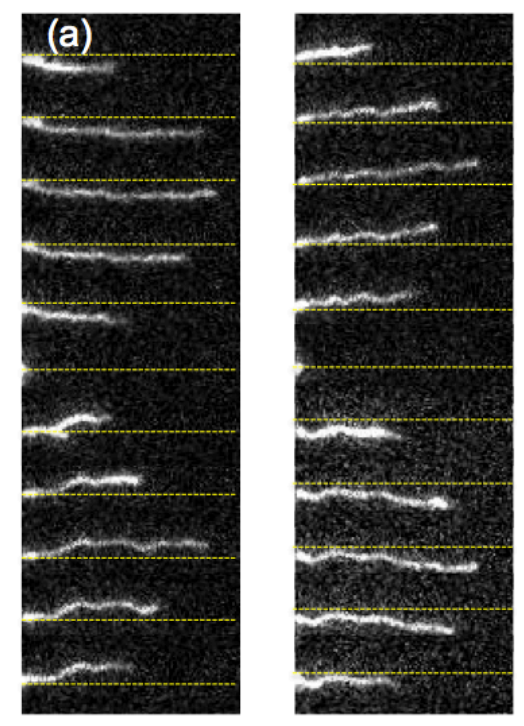

(b)

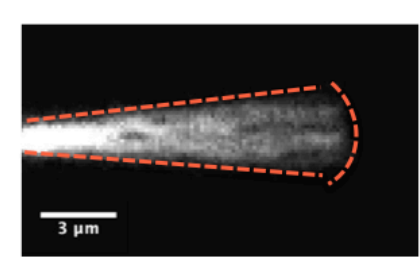

(d)

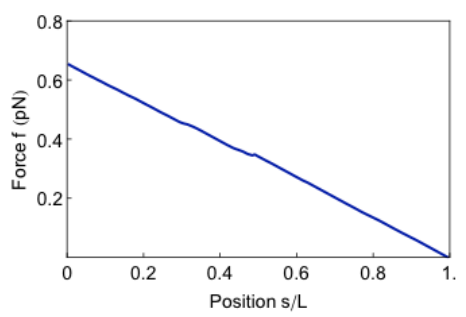

(c)

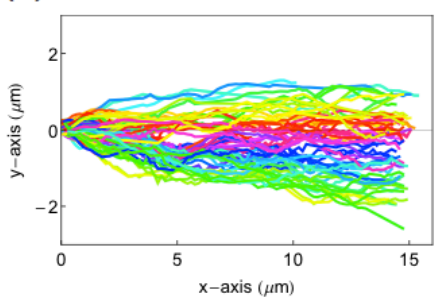

(e)

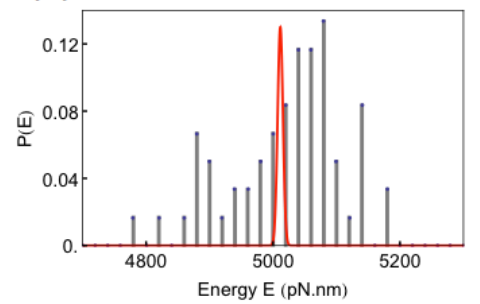

Figure S2 (a) Reptation trajectories of a single DNA molecule in $30 \mathrm{~nm}$ height nanoslits when a field was applied. 70 traces of an elongated DNA superimposed on a single image (b) and their 
contours (c) when a voltage $( \pm 0.03 \mathrm{~V})$ is applied. (d) The force distribution verses position $s / L$ along an elongated tube. (e) The stored electric energy $\left(\varepsilon_{E}\right)$ probability distribution as a function of energy. The red curve indicates thermal fluctuation $\left(k_{B} T\right)$.

\section{Table}

\begin{tabular}{lllll}
\hline \hline$h(\mathrm{~nm})$ & 130 (s.e.m.) & 90 (s.e.m.) & 60 (s.e.m.) & 40 (s.e.m.) \\
\hline$l_{c}(\mathrm{~nm})$ & $48.8 \pm 0.7$ & $76.7 \pm 0.3$ & $79.6 \pm 1.4$ & $88.7 \pm 1.7$ \\
& & & & \\
$x_{0}$ & $0.35 \pm 0.02$ & $0.36 \pm 0.02$ & $0.47 \pm 0.01$ & $0.55 \pm 0.02$ \\
& & & & \\
\hline \hline
\end{tabular}

Table S1: Values of parameters obtained from fitting the eq. (4) for $l_{c}$ and $x_{0}$.

\section{Movies S1-S2:}

Movie S1 and S2 are tethered single DNA molecule stretching in slits height $\mathrm{h}=130 \mathrm{~nm}$ and $30 \mathrm{~nm}$ respectively.

\section{References:}

1. Green, Y.; Eshel, R.; Park, S.; Yossifon, G., Interplay between Nanochannel and Microchannel Resistances. Nano Lett. 2016, 16 (4), 2744-2748.

2. Smith, S. B.; Finzi, L.; Bustamante, C., Direct Mechanical Measurements of the Elasticity of Single DNA-Molecules by Using Magnetic Beads. Science 1992, 258 (5085), 11221126.

3. Perkins, T. T.; Smith, D. E.; Larson, R. G.; Chu, S., Stretching of a Single Tethered Polymer in a Uniform-Flow. Science 1995, 268 (5207), 83-87. 
4. Smith, D. E.; Babcock, H. P.; Chu, S., Single-polymer dynamics in steady shear flow. Science 1999, 283 (5408), 1724-1727.

5. $\quad$ Marko, J. F.; Siggia, E. D., Stretching DNA. Macromolecules 1995, 28 (26), 8759-8770.

6. Bustamante, C.; Marko, J. F.; Siggia, E. D.; Smith, S., Entropic Elasticity of LambdaPhage DNA. Science 1994, 265 (5178), 1599-1600.

7. Manca, F.; Giordano, S.; Palla, P. L.; Cleri, F.; Colombo, L., Theory and Monte Carlo simulations for the stretching of flexible and semiflexible single polymer chains under external fields. J. Chem. Phys. 2012, 137 (24).

8. Yeh, J.-W.; Taloni, A.; Sriram, K. K.; Chen, Y.-L.; Chou, C.-F., Quantitative analysis of reptation of partially extended DNA in sub-30 nm nanoslits. Unpub unpublished (arXiv:1502.05115).

9. Strychalski, E. A.; Levy, S. L.; Craighead, H. G., Diffusion of DNA in Nanoslits. Macromolecules 2008, 41 (20), 7716-7721.

10. Hsieh, C. C.; Balducci, A.; Doyle, P. S., Ionic effects on the equilibrium dynamics of DNA confined in nanoslits. Nano Lett. 2008, 8 (6), 1683-1688.

11. Minatti, E.; Norwood, D. P.; Reed, W. F., Surfactant/polymer assemblies. 2. Polyelectrolyte properties. Macromolecules 1998, 31 (9), 2966-2971. 